ORIGINAL ARTICLE

\title{
Affinity maturation of an anti- $V$ antigen IgG expressed in situ through adenovirus gene delivery confers enhanced protection against Yersinia pestis challenge
}

\author{
TJ Van Blarcom ${ }^{1,2}$, C Sofer-Podesta ${ }^{3}$, J Ang ${ }^{3}$, JL Boyer ${ }^{3}$, RG Crystal ${ }^{3}$ and G Georgiou ${ }^{1,2,4,5}$ \\ ${ }^{1}$ Department of Chemical Engineering, The University of Texas at Austin, Austin, TX, USA; ${ }^{2}$ Institute for Cellular and Molecular \\ Biology, The University of Texas at Austin, Austin, TX, USA; ${ }^{3}$ Department of Genetic Medicine, Weill Cornell Medical College, \\ New York, NY, USA; ${ }^{4}$ Department of Biomedical Engineering, Institute for Cellular and Molecular Biology, The University of \\ Texas at Austin, Austin, TX, USA and ${ }^{5}$ Section of Molecular Genetics and Microbiology, The University of Texas at Austin, Austin, \\ TX, USA
}

Genetic transfer of neutralizing antibodies (Abs) has been shown to confer strong and persistent protection against bacterial and viral infectious agents. Although it is well established that for many exogenous neutralizing Abs increased antigen affinity correlates with protection, the effect of antigen affinity on Abs produced in situ after adenoviral gene transfer has not been examined. The mouse IgG2b monoclonal $A b, 2 C 12.4$, recognizes the Yersinia pestis type III secretion apparatus protein, LcrV ( $V$ antigen), and confers protection in mice when administered as an IgG intraperitoneally or after genetic immunization with engineered, replication-defective serotype 5 human adenovirus (Ad). The $2 C 12.4$ $A b$ was expressed as a single-chain variable fragment ( $s c F v$ ) in Escherichia coli and was shown to display an equilibrium dissociation constant $\left(\mathrm{K}_{D}\right)=3.5 \mathrm{nM}$ by surface plasmon resonance analysis. The $2 \mathrm{C} 12.4 \mathrm{scFv}$ was subjected to random mutagenesis, and variants with increased affinity were isolated by flow cytometry using the anchored periplas- mic expression bacterial display system. After a single round of mutagenesis, variants displaying up to 35-fold lower $\mathrm{K}_{D}$ values $\left(\mathrm{H8}, \mathrm{K}_{D}=100 \mathrm{pM}\right)$ were isolated. The variable domains of the H8 scFv were used to replace those of the parental $2 C 12.4 \mathrm{IgG}$ encoded in the $A d$ vector, $A d \alpha V$, giving rise to $A d \alpha$ V.H8. The two adenoviral vectors resulted in similar titers of anti- $V$ antigen Abs 3 days after immunization, with $10^{9}, 10^{10}$ or $10^{11}$ particle units (pu). After intranasal challenge with 363 $L D_{50}$ (lethal dose, 50\%) of Y. pestis CO92, 54\% of the mice immunized with $10^{10} \mathrm{pu}$ of AdaV.H8 survived through the 14 day end point compared with only 15\% survivors for the group immunized with $A d \alpha V$ expressing the lower-affinity $2 C 12.4$ ( $\mathrm{P}<0.04 ; A d \alpha V$ versus $A d \alpha V . H 8)$. These results indicate that affinity maturation of a neutralizing $A b$ delivered by genetic transfer may confer increased protection not only for $\mathrm{Y}$. pestis challenge but also possibly for other pathogens. Gene Therapy (2010) 17, 913-921; doi:10.1038/gt.2010.42; published online 15 April 2010

Keywords: antibody; affinity maturation; Yersinia pestis; adenovirus gene transfer; protection

\section{Introduction}

Yersinia pestis is the etiological agent of plague and has been responsible for pandemic outbreaks occurring throughout the course of history ${ }^{1}$. Although advances in our current living conditions, public health practices and antibiotic therapies make future pandemics unlikely, outbreaks of plague resulting from biological warfare are a real threat. The features of $Y$. pestis that make it an attractive option for use as a biological weapon include availability of the organism, capacity for aerosol dissemination, potential for spread of secondary cases and the high fatality rate of the pneumonic form of plague. In endemic regions of the world, the bacterium

Correspondence: Professor G Georgiou, Department of Chemical Engineering and Biomedical Engineering, Institute for Cellular and Molecular Biology, The University of Texas at Austin, 1 University Station CO800, Austin, TX 78712, USA. E-mail: gg@che.utexas.edu Received 9 November 2009; revised 23 February 2010; accepted 23 February 2010; published online 15 April 2010 survives by causing chronic disease in animal reservoirs. It is spread among these animals and occasionally to humans predominantly through a flea vector, such as Xenopsylla cheopis. ${ }^{2,3}$ Without prompt antibiotic therapy, $\sim 50 \%$ of bubonic plague infections are fatal and can progress to the more dangerous pneumonic plague. ${ }^{2}$ Respiratory droplets from a pneumonic plague-infected individual promote rapid spread through a susceptible population. Symptoms develop in 1-6 days after infection and the disease progresses rapidly from a flu-like illness to severe pneumonia with cough, chest pain and bloody sputum. To be effective, antibiotic therapy must be administered early. If treatment is delayed more than $24 \mathrm{~h}$ following the onset of symptoms, the fatality rate is high. ${ }^{4}$ In addition, the presence of antibiotic-resistant strains of $Y$. pestis renders antibiotic therapy unreliable. For these reasons, $Y$. pestis is a likely agent to be used as a biological weapon as aerosolized bacteria can confer widespread pneumonic plague. ${ }^{2}$

Of the 11 Yersinia species, only Y. pestis, Y. enterocolitica and $Y$. pseudotuberculosis are human pathogens. $Y$. pestis is 
a gram-negative, nonmotile, non-spore-forming bacterium that replicates intracellularly during the early stages of infection and grows predominantly extracellularly at later stages of the infectious cycle. ${ }^{2}$ At present, no plague vaccine has been approved for use in the United States. Passive immunization with antibodies (Abs) specific for the LcrV protein (V antigen) is an attractive alternative to vaccines, and has been shown to be effective against lethal challenges with $Y$. pestis. ${ }^{1,5-7}$ The $\mathrm{V}$ antigen has a central role in plague pathogenesis. It activates the type III secretion system and thus mediates translocation of effector proteins (Yops) into host macrophages. It is also released from the bacteria and has immunosuppressive functions manifested by increasing levels of the antiinflammatory cytokine interleukin-10 and decreasing levels of tumor necrosis factor- $\alpha(\mathrm{TNF}-\alpha){ }^{2}$ A recently developed anti-V antigen monoclonal antibody (mAb) $2 \mathrm{C} 12.4$ has been shown to confer protection against lethal challenge with intranasally administered $Y$. pestis in a dose-dependent manner. ${ }^{1}$

For several neutralizing Abs, the degree of protection against challenge with pathogen correlates with antigenbinding affinity. ${ }^{8-11}$ For example, although mAbs and $\mathrm{Ab}$ fragments to the protective antigen (PA) of Bacillus anthracis with a equilibrium dissociation constant $\left(K_{\mathrm{D}}\right)=11 \mathrm{nM}$ fail to confer protection against challenge with the holotoxin or with intranasally administered spores, engineered Ab variants displaying 40- to 200-fold higher affinities were protective in different animal models. ${ }^{8,12}$ Notably, protection appeared to be mediated by blocking the ability of PA to bind to its receptor with PEGylated $\mathrm{Ab}$ fragments exhibiting a $K_{\mathrm{D}}=35 \mathrm{pM}$, but lacking an Fc domain, and hence incapable of engaging innate immunity mechanisms of pathogen clearance, were protective. ${ }^{12}$ Engineering Abs with high affinity has been shown to improve protection against other protein toxins and viruses including Botulism, human immunodeficiency virus and human respiratory syncytial virus, and have increased efficacy when targeting inflammatory cytokines such as TNF- $\alpha .^{8-11,13,14}$

In this study, we evaluated whether adenovirus (Ad)mediated delivery of an engineered 2C12.4 IgG, exhibiting markedly increased affinity directed toward the $\mathrm{V}$ antigen can improve protection against $Y$. pestis challenge in mice. ${ }^{1}$ Improvement would be shown by conferring a higher level of protection through immunization with an equivalent dose of a recombinant $\mathrm{Ad}$ encoding the higher-affinity neutralizing $\mathrm{Ab}$. However, the mutations needed to confer higher affinity could have unexpected effects on protein expression from the Ad-delivered IgG DNA or biodistribution of the IgG, thus making it difficult to predict how protection to pathogen challenge might be affected. A single-chain variable fragment ( $\mathrm{scFv}$ ) incorporating the variable heavy chain $\left(\mathrm{V}_{\mathrm{H}}\right)$ and variable light chain $\left(\mathrm{V}_{\mathrm{L}}\right)$ domains of the 2C12.4 IgG was constructed and subjected to affinity maturation by screening a library generated by random mutagenesis. The latter was performed using the Escherichia coli display technique, anchored periplasmic expression (APEx), coupled with fluorescentactivated cell sorting (FACS). ${ }^{15}$ In APEx, the protein library is tethered to the periplasmic side of the inner membrane of the bacterium through fusion to the six N-terminal amino acids of the native lipoprotein NlpA. The resulting $\mathrm{NlpA}-\mathrm{scFv}$ fusion protein is lipidated in vivo and thus becomes attached to the inner membrane. After permeabilization of the E. coli outer membrane by chemical and enzymatic means, the cells are labeled with fluorescent antigen and the most fluorescent cells are isolated by FACS. A single round of random mutagenesis and APEx screening resulted in the isolation of a clone, $\mathrm{H} 8$, displaying a $K_{\mathrm{D}}=100 \mathrm{pM}$ that has a 35-fold higher affinity than the parental 2C12.4 Ab. The $\mathrm{H} 8 \mathrm{~V}_{\mathrm{H}}$ and $\mathrm{V}_{\mathrm{L}}$ domains were used to exchange with those of $2 \mathrm{C} 12.4 \mathrm{IgG}$ in the adenoviral vector $\mathrm{Ad} \alpha \mathrm{V}$, giving rise to $\mathrm{Ad} \alpha \mathrm{V} . \mathrm{H} 8$. We show that delivery of the two vectors in vivo resulted in approximately the same $\mathrm{Ab}$ serum titers, and that mice immunized with $10^{10}$ particle units (pu) of Ad $\alpha$ V.H8 conferred a statistically significant increase in protection relative to the parental $\mathrm{Ad} \alpha \mathrm{V}$.

\section{Results}

\section{Engineering high-affinity anti- $V$ antigen Abs}

Genes encoding the $\mathrm{V}_{\mathrm{H}}$ and kappa light chain $\left(\mathrm{V}_{\mathrm{K}}\right)$ domains of the $Y$. pestis-neutralizing mAb 2C12.4 were used to create $\mathrm{scFv} \mathrm{Ab}$. This scFv gene was cloned into pMoPac16 for soluble expression of the $\mathrm{Ab}$ fragment in the single-chain $\mathrm{Ab}$ (scAb) format. ${ }^{16}$ The scAb format is comprised of an scFv in which the C-terminus of the $\mathrm{V}_{\mathrm{K}}$ domain is fused to the human $\kappa$-constant domain, resulting in improved expression in E. coli without altering the $\mathrm{Ab}$-binding affinity. ${ }^{17}$ Lysates from cells expressing 2C12.4 scAb were confirmed to express fulllength protein by western blotting and displayed binding activity, as determined by enzyme-linked immunosorbent assay (ELISA) using purified $Y$. pestis $\mathrm{V}$ antigen.

The gene encoding the scFv variant of $2 \mathrm{C} 12.4$ was subjected to one round of random mutagenesis by errorprone PCR. Amplified DNA was cloned into pFLAGAPEx and the ligation product was transformed into E. coli Jude- 1 cells yielding $>2 \times 10^{6}$ independent transformants. DNA sequencing of 10 clones selected at random revealed an average of $1.75 \%$ nucleotide substitutions with a standard deviation of $0.75 \%$. Cells were grown in liquid culture, protein expression was induced and $4 \mathrm{~h}$ later, cells were harvested and converted into spheroplasts. The spheroplasted cells were then labeled with anti-FLAG R-phycoerythrin (PE) and V antigen-BODIPY (dipyrrometheneboron difluoride) to determine full-length $\mathrm{scFv}$ expression levels and antigen binding, respectively (Figure 1a). A total of $8 \times 10^{7}$ cells (40-fold library coverage) were subjected to highthroughput FACS, and the top $2 \%$ events in terms of PE and BODIPY fluorescence emissions were collected (Figure 1b). The collected spheroplasts were immediately resorted as above without additional labeling to maximize the isolation of Abs with slow dissociation rate constants toward $\mathrm{V}$ antigen. ${ }^{15}$ The $\mathrm{scFv}$ genes in the spheroplasts collected during the resort were amplified by PCR, and the DNA was subcloned back into the pFLAG-APEx vector. After transformation, the cells were subjected to two additional rounds of sorting using increasingly stringent collection criteria, namely, a higher fluorescence threshold. After the third round of sorting, the number of events falling within the window used for the first round increased from 2 to $23 \%$ (Figure 1b). 

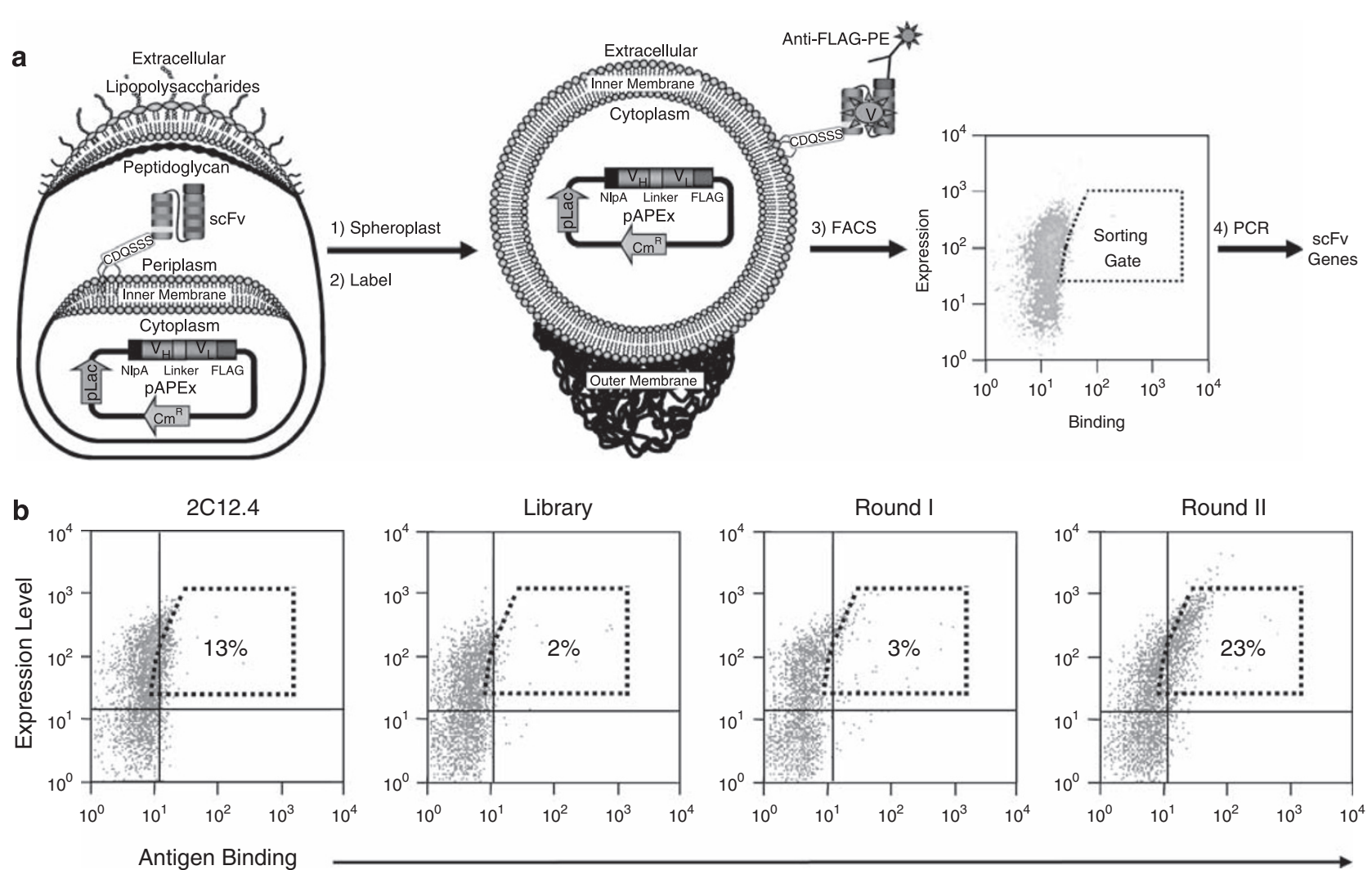

Figure 1 Affinity maturation of the anti-V antigen $\mathrm{scFv} 2 \mathrm{C} 12.4$ using anchored periplasmic expression (APEx). (a) Schematic diagram of APEx (b) FACS analysis of E. coli spheroplasts expressing $2 \mathrm{C} 12.4 \mathrm{scFv}$, the library of random mutants and the populations recovered after two rounds of FACS. Spheroplasts were labeled with $500 \mathrm{nM} \mathrm{V}$ antigen-BODIPY and $5 \mu \mathrm{g} \mathrm{ml}{ }^{-1}$ Anti-FLAG-PE. The dashed box represents the gate used to isolate cells by FACS from the original library. The percentage of the spheroplast in this gate for each population is indicated.

Table 1 Kinetic analysis of anti-V antigen antibody fragments

\begin{tabular}{llccrr}
\hline Antibody & $\mathrm{k}_{\text {on }}\left(\mathrm{M}^{-1} \mathrm{~S}^{-1}\right)^{\mathrm{a}}$ & $\mathrm{k}_{\text {off }}\left(\mathrm{s}^{-1}\right)^{\mathrm{a}}$ & $\mathrm{K}_{D}(p M)$ & Affinity improvement $^{\mathrm{b}}$ & Expression improvement $^{\mathrm{c}}$ \\
\hline $2 \mathrm{C} 12.4$ & $9.2(0.005) \times 10^{4}$ & $3.2(0.005) \times 10^{-4}$ & 3500 & $1 \times$ & $1 \times$ \\
H8 & $1.2(0.0009) \times 10^{5}$ & $1.2(0.08) \times 10^{-5}$ & 100 & $35 \times$ & $4 \times$ \\
E4 & $1.0(0.0007) \times 10^{5}$ & $1.8(0.07) \times 10^{-5}$ & 180 & $20 \times$ & $5 \times$ \\
F4 & $1.2(0.0007) \times 10^{5}$ & $2.6(0.06) \times 10^{-5}$ & 220 & $16 \times$ & $3 \times$ \\
G8 & $9.8(0.007) \times 10^{4}$ & $2.4(0.07) \times 10^{-5}$ & 250 & $14 \times$ & $2 \times$ \\
\hline
\end{tabular}

${ }^{a}$ Global fit (and standard error for the fit) of triplicate injections at four different antibody concentrations.

${ }^{\mathrm{b}}$ Affinity improvement relative to the parental antibody $2 \mathrm{C} 12.4$.

'Yield of purified scAb $\left(\mathrm{mg} \mathrm{l}^{-1} ; \mathrm{A}_{600}\right)$ compared with 2C12.4.

The scFv gene pool from the third round of sorting was amplified by PCR and cloned into pMoPac16 for expression in the scAb format. Surface plasmon resonance (SPR) was used to rank order the dissociation rate constants of individual clones (Supplementary Figure 1). Of the 72 colonies tested, 41 (57\%) expressed scAbs that displayed significant binding toward $\mathrm{V}$ antigen immobilized on the Biacore chip (GE Healthcare, Uppsala, Sweden). Of these 41 clones, four clones exhibited dissociation rate constants at least 10 -fold slower than those expressing the parental $\mathrm{Ab}$ (Supplementary Figure 1e and f).

These four scAbs, as well as the parental 2C12.4 and the digoxin specific $26.10 \mathrm{scAbs}$, were expressed at the $500 \mathrm{ml}$ scale in shake flasks, the periplasmic fraction was isolated by osmotic shock and monomeric scAb proteins were purified by immobilized metal affinity chromatography and size-exclusion fast protein liquid chromatography. Consistent with the isolation of the respective clones on the basis of increased PE fluorescence, the protein yields for all four scAbs was higher than that of the parental 2C12.4 scAb (Table 1). All Abs exhibited at least 10-fold lower $K_{\mathrm{D}}$ relative to the parental $2 \mathrm{C} 12.4 \mathrm{scAb}$ (Table 1), which is consistent with the measurements obtained using crude cell lysates (Supplementary Figure 1f). The highest-affinity clone, H8, exhibited an affinity of $100 \mathrm{pM}$ that translates to a 35 -fold improvement compared with the parental $\mathrm{Ab}$ 2 C12.4. The improved affinity for all clones was almost exclusively the result of a decrease in the dissociation rate constant, whereas the association rate constant remained essentially unchanged. The four variants, H8, 
Table 2 Sequence analysis of anti-V antigen antibody fragments

\begin{tabular}{|c|c|c|c|c|c|c|c|c|c|c|c|c|c|c|c|c|c|c|}
\hline \multirow[b]{3}{*}{ Clone } & \multicolumn{9}{|c|}{ Variable heavy chain ${ }^{a}$} & \multicolumn{9}{|c|}{ Variable light chain ${ }^{a}$} \\
\hline & \multicolumn{2}{|c|}{$F W 1$} & \multirow{2}{*}{$\begin{array}{c}\text { CDR1 } \\
32\end{array}$} & \multicolumn{2}{|c|}{$F W 2$} & \multicolumn{2}{|c|}{$C D R 2$} & \multicolumn{2}{|c|}{ FW3 } & \multicolumn{2}{|c|}{$F W 1$} & \multicolumn{2}{|c|}{ CDR1 } & \multirow{2}{*}{$\begin{array}{c}F W 2 \\
39\end{array}$} & \multirow{2}{*}{$\begin{array}{c}C D R 2 \\
53\end{array}$} & \multicolumn{2}{|c|}{ FW3 } & \multirow{2}{*}{$\begin{array}{c}C D R \\
97\end{array}$} \\
\hline & 3 & 11 & & 45 & 49 & 51 & 65 & 67 & 82 & 1 & 5 & 24 & 32 & & & 72 & 84 & \\
\hline $2 C 12.4$ & $T$ & $I$ & $S$ & $L$ & $L$ & $I$ & $S$ & $L$ & $I$ & $D$ & $T$ & $R$ & Y & K & $N$ & $T$ & $A$ & $T$ \\
\hline $\mathrm{H} 8$ & & & & & S & $\mathrm{V}$ & & & & $\mathrm{N}$ & $\mathrm{S}$ & $\mathrm{K}$ & $\mathrm{F}$ & & & & & $\mathrm{R}$ \\
\hline E4 & & $\mathrm{V}$ & $\mathrm{P}$ & & S & & & & & & & & $\mathrm{F}$ & $\mathrm{R}$ & $S$ & & $\mathrm{~T}$ & \\
\hline F4 & A & & & $\mathrm{P}$ & $S$ & & & $\mathrm{~V}$ & & $\mathrm{~N}$ & $S$ & $\mathrm{~K}$ & $\mathrm{~F}$ & & & & & $\mathrm{R}$ \\
\hline G8 & & & & & S & & G & & $\mathrm{T}$ & $\mathrm{N}$ & $S$ & $\mathrm{~K}$ & $\mathrm{~F}$ & & & $\mathrm{~A}$ & & $\mathrm{R}$ \\
\hline
\end{tabular}

Abbreviations: CDR, complementary determining region; FW, framework.

${ }^{a}$ Amino acid substitutions based on Kabat numbering.

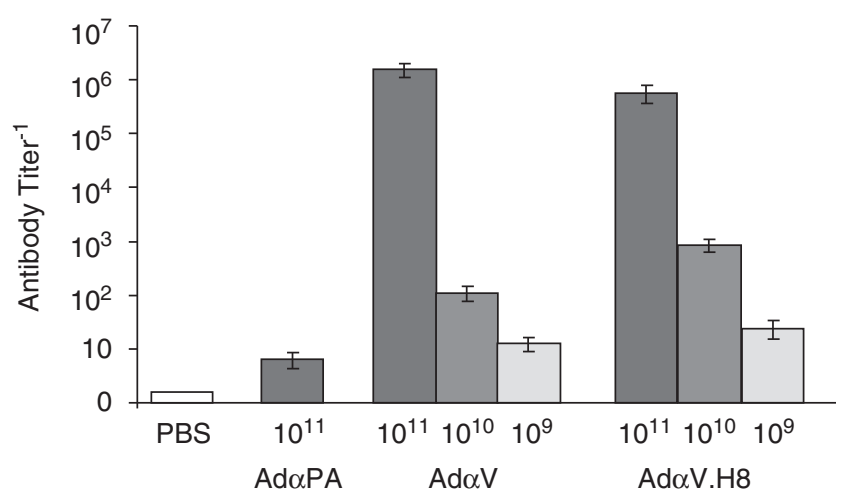

Figure 2 Anti-V antigen IgG titers in mice after immunization with Ad vectors. C57BL $/ 6$ mice ( $n=13$ per group for PBS, $\mathrm{Ad} \alpha \mathrm{PA}, \mathrm{Ad} \alpha \mathrm{V}$ and $\mathrm{Ad} \alpha \mathrm{V} . \mathrm{H} 8$ ) were intravenously administered with the indicated Ad vectors or PBS. Serum anti-V antigen antibody titers were measured 3 days after immunization by ELISA. PBS, negative control; Ad $\alpha \mathrm{PA}$, vector encoding an IgG specific for the $B$. anthracis PA; $\mathrm{Ad} \alpha \mathrm{V}$, vector encoding the $2 \mathrm{C} 12.4 \mathrm{IgG}$; $\mathrm{Ad} \alpha \mathrm{V} . \mathrm{H} 8$, vector encoding the 2C12.4 IgG mutant $\mathrm{H} 8$.

E4, F4 and G8, contained either 7 or 9 amino acid mutations compared with the parental $\mathrm{Ab}$ (Table 2), of which two were common: L49S in framework 2 of the $\mathrm{V}_{\mathrm{H}}$, which is immediately before complementary determining region 2 , and $\mathrm{Y} 32 \mathrm{~F}$ in complementary determining region 1 of the light chain. ${ }^{18}$ Further, three of the variants contained an additional four common mutations (D1N, T5S, R24K and T97R) in the light chain.

\section{Genetic immunization using IgG-encoding Ad vectors and challenge with $\mathrm{Y}$. pestis}

The $\mathrm{V}_{\mathrm{H}}$ and $\mathrm{V}_{\mathrm{K}}$ domains of the $2 \mathrm{C} 12.4$ mouse IgG2b Ab were replaced with those from the highest-affinity $\mathrm{Ab}$ variant, $\mathrm{H} 8$, and engineered into a replication-defective Ad serotype 5 E1-E3 gene transfer vector to create Ad $\alpha$ V.H8. ${ }^{1}$ Mice were immunized with varying doses of $\mathrm{Ad} \alpha \mathrm{V}$ and Ad $\alpha \mathrm{V} . \mathrm{H} 8\left(10^{9}-10^{11} \mathrm{pu}\right)$ or, as controls, the highest dose $\left(10^{11} \mathrm{pu}\right)$ of Ad $\alpha \mathrm{PA}$, an Ad vector encoding a mouse IgG $\mathrm{mAb}$ specific for the $B$. anthracis $\mathrm{PA}$, or phosphate-buffered saline (PBS). Serum anti-V antigen $\mathrm{Ab}$ titers were measured by ELISA 3 days after immunizations (Figure 2). A similar dose-dependent response was observed for both $\mathrm{Ad} \alpha \mathrm{V}$ and Ad $\alpha \mathrm{V}$.H8. The titers for equivalent immunization levels were within 10-fold for both $\mathrm{Ad} \alpha \mathrm{V}$ and $\mathrm{Ad} \alpha \mathrm{V} . \mathrm{H} 8$. Both Ad $\alpha \mathrm{PA}$ and PBS controls did not result in any measurable anti-V antigen $\mathrm{Ab}$ titers. At 3 days after immunization, the mice were challenged intranasally with $363 \mathrm{LD}_{50}$ (lethal dose, $50 \%$ ) of the fully virulent $Y$. pestis CO92 and survival was monitored for 2 weeks (Figure 3). All mice immunized with the highest dosage ( $10^{11} \mathrm{pu}$ ) of $\mathrm{Ad} \alpha \mathrm{V}$ and $\mathrm{Ad} \alpha \mathrm{V} . \mathrm{H} 8$ survived (Figure 4). However, at a 10-fold lower dose $\left(10^{10} \mathrm{pu}\right)$, only $15 \%$ of the mice immunized with $\mathrm{Ad} \alpha \mathrm{V}$ survived, whereas $54 \%$ of the mice immunized with this dose of $\mathrm{Ad} \alpha \mathrm{V} . \mathrm{H} 8$ survived $(P<0.04 ; \mathrm{Ad} \alpha \mathrm{V}$ versus Ad $\alpha$ V.H8). In addition, $15 \%$ of the mice immunized with an additional 10 -fold lower dose $\left(10^{9} \mathrm{pu}\right)$ of $\mathrm{Ad} \alpha \mathrm{V} . \mathrm{H} 8$ survived, but none survived with the same dosage level of $\operatorname{Ad} \alpha \mathrm{V}(P<0.1 ; \mathrm{Ad} \alpha \mathrm{V}$ versus $\mathrm{Ad} \alpha \mathrm{V} . \mathrm{H} 8)$.

\section{Discussion}

Passive immunization with engineered Abs displaying enhanced antigen affinity has been shown to increase the neutralization efficacy of a number of bacterial or viral pathogens and toxins. ${ }^{8-11,13,14}$ However, to the best of our knowledge, neither the effect of antigen affinity for Abs expressed in situ after viral transfer of the $\mathrm{Ab}$ gene nor the relationship between $\mathrm{V}$ antigen affinity and protection to the potential biowarfare agent $Y$. pestis, have been examined. Here, we found that increasing the affinity of the $Y$. pestis-neutralizing $\mathrm{Ab} 2 \mathrm{C} 12.4$ from $3.5 \mathrm{nM}$ to $100 \mathrm{pM}$ (35-fold) could confer significant protection against a lethal challenge with $Y$. pestis at a 10-fold lower dose of a recombinant Ad encoding the neutralizing $\mathrm{Ab}$.

The affinities of Abs generated by the natural immune system are constrained by the kinetics of in vivo selection. ${ }^{19}$ Most high-affinity mouse mAbs display nanomolar affinities and this is the case for the anti- $\mathrm{V}$ antigen $\mathrm{Ab} 2 \mathrm{C} 12.4$, which displays a $K_{\mathrm{D}}=3.5 \mathrm{nM}$ by SPR. Combinatorial mutagenesis and library screening by phage display or other high-throughput techniques have been used for affinity maturation of the variable domains of mAbs. ${ }^{8-11,13,14}$ In earlier studies, we used random mutagenesis and screening of E. coli displayed libraries by APEx to isolate picomolar affinity $\mathrm{Abs}$ to the B. anthracis PA, which in turn proved to have markedly improved neutralization potency in vitro and in various animal models. ${ }^{8,12,20}$ Key advantages of this strategy are the ease with which libraries of random mutants can be constructed in E. coli and screened by FACS. FACS is 

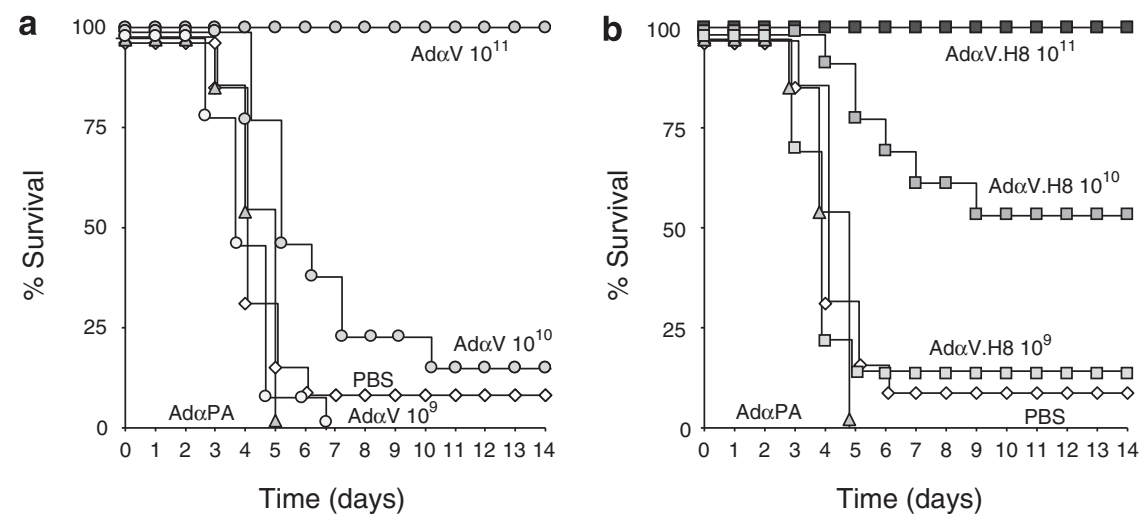

Figure 3 Survival of mice challenged with Y. pestis after prophylactic administration of Ad vectors. At 3 days after intravenous administration of Ad vectors or PBS, C57BL/6 mice $(n=13$ per group for PBS, Ad $\alpha \mathrm{PA}, \mathrm{Ad} \alpha \mathrm{V}$ and $\mathrm{Ad} \alpha \mathrm{V}$.H8) were challenged with a lethal dose of $Y$. pestis intranasally. Survival of the mice was monitored for 14 days after challenge. (a) Immunization with Ad $\alpha \mathrm{V}$ compared with Ad $\alpha$ PA and PBS. (b) Immunization with Ad $\alpha$ V.H8 compared with Ad $\alpha$ PA and PBS.

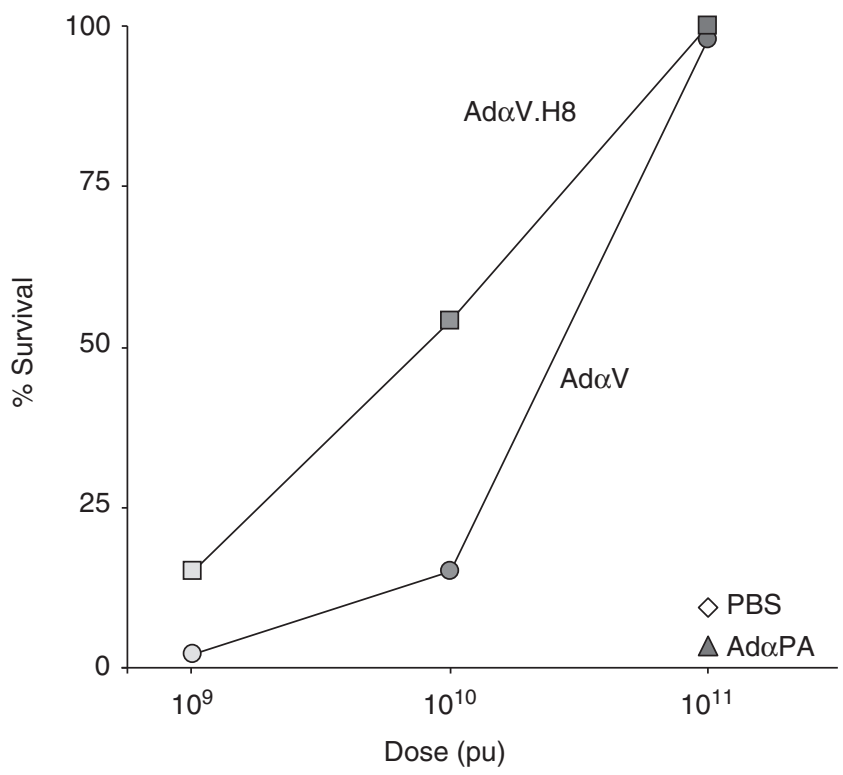

Figure 4 Net survival of mice challenged with $Y$. pestis after prophylactic administration of Ad vectors. Percentage of C57BL/6 mice $(n=13$ per group for PBS, Ad $\alpha \mathrm{PA}, \mathrm{Ad} \alpha \mathrm{V}$ and $\mathrm{Ad} \alpha \mathrm{V} . \mathrm{H} 8)$ surviving 14 days after intranasal challenge with a lethal dose of Y. pestis.

a high-throughput screening technique that enables real-time quantitative multi-parameter analysis on a cell by cell basis. Therefore, multiple properties can be monitored simultaneously to specifically isolate Abs with desired characteristics. In this work, Ab fragments were isolated from a library on the basis of both antigen affinity and expression level (Figure 1). Subsequently, clones isolated from the last round of FACS were rankordered by comparing the dissociation rate constants in crude lysates (Supplementary Figure 1). This approach significantly expedited the analysis of the clones obtained after screening, a process that often represents the rate-limiting step in $\mathrm{Ab}$ affinity maturation. Thus, a single round of mutagenesis followed by three rounds of FACS screening resulted in the isolation of Abs displaying affinities in the $100 \mathrm{pM}$ range and also higher expression yields in E. coli (Figure 1b, Table 1). In addition, the conversion of these monovalent $\mathrm{Ab}$ fragments to full-length Abs can further enhance their potency owing to the avidity associated with these bivalent molecules, leading to an even higher apparent affinity.

Genetic delivery of full-length Abs in vivo has been carried out using Ad, adeno-associated virus and vaccinia virus vectors, ${ }^{21-23}$ and it is an attractive alternative to the administration of $\mathrm{Ab}$ protein, which requires production, purification and formulation before injection into the patient. ${ }^{22,24}$ The administration of Abs as injectable therapeutics is complicated by bioavailability issues and by the potential for increased immunogenicity resulting from large dosages required for therapeutic purposes and the ensuing aggregation issues. ${ }^{25}$ A common side effect is infusion reactions with symptoms ranging from mild to life-threatening and typically occur near the time of administration. ${ }^{26}$ After infusions, complicated pharmacokinetics result in a continual decrease in serum concentrations that effects the bioavailability and the pharmacodynamic properties of the therapeutic $A b{ }^{25,27}$ However, genetic delivery can provide immediate and sustained serum levels for several months from a single treatment with both Ad and adeno-associated virus vectors encoding the same $\mathrm{mAb} .^{28}$ This approach has been shown using a neutralizing $\mathrm{Ab}$, and it offered almost immediate protection against anthrax toxin challenge in vivo, which was sustained for 6 months. ${ }^{29}$ This approach circumvents many of the issues associated with conventional methods and has the additional benefits of increasing patient quality of life and protecting soldiers entering combat for extended periods.

Here, we show that the affinity of Abs produced in situ after adenoviral gene transfer correlates with protection. Although human Ad serotype 5 has been effectively used in a variety of animal models for genetic delivery, it has limited use because $\sim 35-50 \%$ of humans have preexisting neutralizing Abs against it. ${ }^{30}$ However, several nonhuman primate Ad vectors for which no natural immunity exists in humans have been described and can be deployed to circumvent the neutralization or clearance of the vector. ${ }^{28}$ There are many pathogens to which an effective vaccine has yet to be produced, and 
neutralizing Abs remain the only means for prophylaxis and therapy. The potency of neutralizing Abs can benefit from engineering their affinity, stability and neutralization potency (for instance, through mutations that increase $\mathrm{Ab}$-dependent cell-mediated cytotoxicity ${ }^{31}$ ). The in situ synthesis of optimized neutralizing Abs by lowimmunogenicity genetic delivery vectors could represent a promising alternative for the treatment of a number of infectious diseases.

\section{Materials and methods}

\section{Bacterial strains and plasmid vectors}

E. coli Jude-1 (E. coli DH10B F ${ }^{-}$mcrA $\Delta$ (mrr-hsdRMSmcrBC) $\phi 80 \mathrm{~d} l a c Z \Delta \mathrm{M} 15 \Delta$ lacX74 recA1 araD139 $\Delta$ (ara leu)7697 gall galK rpsL endA1 nup G harboring an $\mathrm{F}^{\prime}$ from E. coli XL1-blue introduced by conjugation) was used for all $\mathrm{Ab}$ engineering experiments. Plasmid pMoPac16 was used for soluble scAb fragment expression and has been previously described. ${ }^{16}$ Plasmids pAPEx1 was used for E. coli display of soluble scFv Abs. ${ }^{15}$ pFLAG-APEx was constructed by replacing the polyhistidine and c-myc sequences in pAPEx1 through BamHI and NotI restriction sites with the FLAG peptide epitope sequence amplified by PCR using primers TVB100 (5'-GTCGCT GCGGCCGCAGATTACAAAGACGACGATGACAAGTA GTGATATCGCAAGCTTGACC-3') and TVB101 (5'-CAG CGAGGATCCGTGACGCAGTAGCGGTAAACGGC-3').

\section{Protein expression and purification}

Recombinant $\mathrm{V}$ antigen was expressed and purified as previously described. ${ }^{1,32}$ Monomeric scAbs were expressed and purified according to the published procedures. ${ }^{15,16}$ Briefly, E. coli Jude-1 containing pMoPac16 derivatives encoding the desired $\mathrm{scAb}$ genes were streaked from frozen stocks onto agar plates containing Luria-Burtani Miller (LB) medium (Becton Dickinson Difco, Sparks, MD, USA) supplemented with $2 \%$ (wt/vol) glucose (2\% glc) and $200 \mu \mathrm{g} \mathrm{ml}^{-1}$ ampicillin (Amp200) $\left(20 \mathrm{~h}, 25^{\circ} \mathrm{C}\right)$. Individual colonies were cultured in $2 \mathrm{ml}$ of Terrific Broth (TB) medium (Becton Dickinson Difco) $+2 \%$ glc + Amp200 (8 h, 250 r.p.m., $\left.30{ }^{\circ} \mathrm{C}\right)$ then $1 \mathrm{ml}$ was used to inoculate $500 \mathrm{ml}$ of TB medium $+2 \%$ glc + Amp200 (250 r.p.m., $30^{\circ} \mathrm{C}$ ). After overnight growth, cells were pelleted by centrifugation (15 min, $4400 \times g, 4{ }^{\circ} \mathrm{C}$ ) and resuspended in $500 \mathrm{ml}$ of $\mathrm{TB}$ medium+Amp200+1 mM isopropyl- $\beta$-D-thiogalactopyranoside (IPTG) (Sigma-Aldrich, St. Louis, MO, USA) to induce protein expression. After $4 \mathrm{~h}$ of incubation at $25^{\circ} \mathrm{C}$, the cells were pelleted by centrifugation ( $15 \mathrm{~min}$, $4400 \times g, 4{ }^{\circ} \mathrm{C}$ ) and the periplasmic fraction from the cell pellet was isolated by osmotic shock according to the published procedures. $^{16} \mathrm{scAb}$ proteins were purified from the shockate by immobilized metal affinity chromatography using Ni-NTA agarose on the basis of the manufacturer's protocol (Qiagen, Hilden, Germany), and monomeric scAbs were further isolated by size-exclusion fast protein liquid chromatography on a Superdex 200 column (GE Healthcare) using HEPES (4-(2-hydroxyethyl)-1-piperazineethanesulfonic acid)-buffered saline with surfactant P20 (HBS-P) (GE Healthcare). Proteins were quantified on the basis of the absorbance $\left(\mathrm{A}_{280}\right)$ measured using a NanoDrop 1000 (Thermo Fisher Scientific, Wilmington, DE, USA) and the appropriate extinction coefficients calculated using Protein Calculator (http:// www.scripps.edu/ cdputnam/protcalc.html, Putnam Lab at The Scripps Research Institute, La Jolla, CA, USA). Relative concentrations were verified and purity determined by sodium dodecyl sulfate polyacrylamide gel electrophoresis using a 4-20\% gel (NuSep, Lawrenceville, GA, USA) stained with GelCode Blue (Thermo Fisher Scientific, Rockford, IL, USA). All scAbs used in this work were at least $90 \%$ pure.

\section{Anti-V antigen $A b$ fragment cloning, expression and analysis}

Genes encoding the $V_{H}$ and the $V_{K}$ domains of anti-V antigen 2C12.4 IgG were amplified by PCR from the previously described Ad vector plasmid $\mathrm{DNA}^{1}$ using primers TVB102 (5'-GCAGCGAGGCCCAGCCGGCCATG GCGCAGGTAACTCTGAAAGAGTCTG-3') and TVB103 (5'-AGAGCCGCCGCCGCCGCTACCACCACCACCAGAA CCACCACCACCTGAGGAGACTGTGAGAGTGGTG-3') for $\mathrm{V}_{\mathrm{H}}$ and TVB104 (5'-GGTGGTGGTGGTAGCGGCGGCG GCGGCTCTGGCGGCGGCGGCTCCGACATTGTGCTGAC ACAGTCG-3') and TVB105 (5'-CGAATTCGGCCCCCGAG GCCCGTTTTACTTCCAGCTTGGTC-3') for $\mathrm{V}_{\mathrm{K}} \cdot{ }^{1}$ The resulting PCR products were combined by overlap extension PCR and cloned into pMoPac16 through the non-compatible SfiI restriction sites for soluble expression as a scAb. The resulting ligation product was transformed into E. coli Jude-1, and $\mathrm{scAb}$ expression was performed as previously described. ${ }^{16,33}$ Briefly, individual colonies were cultured overnight in $2 \mathrm{ml}$ of TB medium $+2 \%$ glc + Amp200 at $30{ }^{\circ} \mathrm{C}$. After overnight growth, cells were pelleted by centrifugation $(10 \mathrm{~min}$ at $4600 \times g, 4{ }^{\circ} \mathrm{C}$ ) and resuspended in $2 \mathrm{ml}$ of TB mediu$\mathrm{m}+$ Amp200+1 mM IPTG to induce protein expression. After $4 \mathrm{~h}$ incubation at $25^{\circ} \mathrm{C}, 1 \mathrm{ml}$ of cells were pelleted by centrifugation $\left(10 \mathrm{~min}\right.$ at $4600 \times g, 4^{\circ} \mathrm{C}$ ), and the cell pellet was resuspended in $1 \mathrm{ml}$ of lysis buffer that consisted of BugBuster HT Protein Extraction Reagent (Novagen, Madison, WI, USA) diluted in the ratio 1:4 in PBS. ${ }^{34}$ The cells were incubated in lysis buffer while rotating on an inverter (30 min, 60 r.p.m., $25^{\circ} \mathrm{C}$ ) and then the insoluble fraction was removed by centrifugation $\left(1 \mathrm{~min}, 10000 \times g, 4{ }^{\circ} \mathrm{C}\right)$.

The soluble fraction was analyzed for the presence of full-length scAb specific for the $\mathrm{V}$ antigen by western blot analysis and ELISA. Western blot analysis with anti-polyhistidine (anti-His) peroxidase (HRP) conjugate (Sigma-Aldrich) was performed as previously described. ${ }^{35}$ ELISA was performed by coating Costar high-binding 96-well EIA/RIA plates (Corning, Corning, $\mathrm{NY}$, USA) overnight at $4{ }^{\circ} \mathrm{C}$ with $50 \mu \mathrm{l}$ of $5 \mu \mathrm{g} \mathrm{ml}{ }^{-1}$ recombinant $\mathrm{V}$ antigen or bovine serum albumin in PBS. The plates were washed three times with PBS, followed by blocking with $400 \mu \mathrm{l}$ PBS supplemented with $2 \%$ milk for $4 \mathrm{~h}$ at room temperature. Samples were diluted in the ratio 1:1 in PBS with 2\% milk and incubated for $1 \mathrm{~h}$ at room temperature. The plates were washed three times with PBS and an additional three times with PBS containing $0.1 \%$ Tween-20. Immunocomplexes were detected using an anti-human $\kappa$-light chain (anti-HuCк) polyclonal serum HRP conjugate (Sigma-Aldrich) applied at a 1:10 000 dilution and incubated for $30 \mathrm{~min}$ at room temperature. The plates were washed as described above and developed using the chromogenic HRP substrate $\mathrm{TMB}+$ (Dako, Glostrup, Denmark), as 
described by the manufacturer. The $\mathrm{A}_{450}$ was measured with a mircroplate reader (BioTek, Winooski, VT, USA).

\section{Isolation of high-affinity variants of the $2 C 12.4 \mathrm{scFV}$}

The anti-V antigen $2 \mathrm{C} 12.4 \mathrm{scFv}$ and the anti-digoxin 26.10 $\mathrm{scFv}$ were cloned into pAPEx1 and pFLAG-APEx through the non-compatible SfiI restriction sites. ${ }^{15}$ Purified recombinant $\mathrm{V}$ antigen was conjugated to BODIPY using 6-((4,4-difluoro-5,7-dimethyl-4-bora-3a,4a-diaza-sindacene-3-propionyl)amino) hexanoic acid succinimidyl ester (Invitrogen, Carlsbad, CA, USA) at a molar ratio of $1: 5$, as described by the manufacturer. Free BODIPY FL-X SE was removed using a NAP-10 gel filtration column, as described by the manufacturer (GE Healthcare). The extent of conjugation was determined on the basis of the ratio of $A_{280}$ and $A_{504}$ measured using a NanoDrop 1000 and the appropriate extinction coefficients and correction factors as recommended by the manufacturer (http://www.scripps. edu/ cdputnam/protcalc.html, Putnam Lab at The Scripps Research Institute; Invitrogen).

The anti-V antigen $2 \mathrm{C} 12.4 \mathrm{scFv}$ was subjected to random mutagenesis by error-prone $\mathrm{PCR}^{36}$ the amplified DNA was cloned into pFLAG-APEx through the non-compatible SfiI restriction sites, and the ligation product was transformed into electrocompetent $E$. coli Jude-1. ${ }^{15}$ Cells were cultured on agar plates containing $\mathrm{LB}$ medium $+2 \%$ glc $+30 \mu \mathrm{g} \mathrm{ml}^{-1}$ chloramphenicol $(\mathrm{Cm} 30)$ $\left(22 \mathrm{~h}, 25{ }^{\circ} \mathrm{C}\right)$. Cells were transferred to LB medium $+2 \%$ glc $+15 \%$ glycerol $(\mathrm{v} / \mathrm{v})$, to $\mathrm{A}_{600} \sim 10$ and then stored at $-80{ }^{\circ} \mathrm{C}$. Frozen cell stocks were thawed on ice and subcultured to $\mathrm{A}_{600}=0.1$ in $\mathrm{TB}$ medium $+2 \%$ glc $+\mathrm{Cm} 30$ grown up at $37{ }^{\circ} \mathrm{C}$, to $\mathrm{A}_{600} \sim 0.8-1.2$, cooled for $30 \mathrm{~min}$ to $25{ }^{\circ} \mathrm{C}$ and protein expression was induced with the addition of $1 \mathrm{mM}$ IPTG. After $4 \mathrm{~h}$ induction at $25^{\circ} \mathrm{C}, 1 \mathrm{ml}$ of cells at $\mathrm{A}_{600}=5.0$ were converted to spheroplasts using Tris/sucrose/EDTA/lysozyme as previously described. ${ }^{15,34}$ The spheroplasted cells were first labeled with $5 \mu \mathrm{g} \mathrm{ml}^{-1}$ PhycoLink anti-FLAG R-phycoerythrin (Prozyme, San Leandro, CA, USA) in PBS supplemented with $1 \%$ bovine serum albumin (PBSB) (30 min, 150 r.p.m., $\left.25^{\circ} \mathrm{C}\right)$. The spheroplasts were washed with PBSB and incubated with $500 \mathrm{nM} \mathrm{V}$ antigen conjugated to BODIPY (V antigen-BODIPY) in PBSB (1 h, 150 r.p.m., $\left.25^{\circ} \mathrm{C}\right)$. Labeled spheroplasts were washed with PBSB and analyzed on a FACSAria (Becton Dickinson Biosciences, San Jose, CA, USA) droplet deflection flow cytometer by exciting with a 488-nm laser and measuring the fluorescence emission spectrum of BODIPY and PE with $530 / 40$ and $570 / 40$ band-pass filters, respectively. Fluorescence compensation was performed using the APEx controls as previously described. ${ }^{34}$ The library population was gated to avoid aggregates, as determined by the forward scatter and side scatter parameters, and the brightest $1-2 \%$ of the population based on both the BODIPY and PE emission spectrums was collected. ${ }^{15,34}$ The collected spheroplasts were immediately resorted, and the $s c F v$ genes in the resort solution were amplified by PCR using primers BRH06 (5'-GCGGATAACAATT TCACACAGG-3') and AHX89 (5'-CGCAGTAGCGGT AAACGGC- $\left.3^{\prime}\right){ }^{15}$ The amplified DNA was cloned into pFLAG-APEx through the non-compatible SfiI restriction sites, and the ligation mixture was transformed into electrocompetent $E$. coli Jude- 1 . At least 10 -fold excess of colonies relative to the number of events in the resort were obtained. Colonies were scraped from the agar plates into liquid media as explained above and subjected to an additional two rounds of sorting, exactly as described above.

The $\mathrm{scFv}$ genes from the third round of sorting were subcloned into pMoPac16, and the ligation product was transformed into E. coli Jude-1 for expression of soluble scAb similar to above. ${ }^{15}$ In addition, E. coli Jude-1 containing pMoPac16 encoding 2C12.4 scAb was streaked from a frozen stock onto an agar plate containing LB medium+2\% glc+Amp200. Further, the antidigoxin $26.10 \mathrm{scFv}$ was cloned into pMoPac16 through the non-compatible SfiI restriction sites, and the ligation product was transformed into E. coli Jude-1. A total of 72 individual colonies from round III, 8 colonies of $2 \mathrm{C} 12.4$ $\mathrm{scAb}$ and 4 colonies of $26.10 \mathrm{scAb}$ were picked and cultured overnight in sterile Costar round-bottomed 96-well microtiter plates (Corning) containing $200 \mu \mathrm{l}$ of TB medium $+2 \%$ glc + Amp200 on a microtiter plate shaker. After overnight growth (150 r.p.m., $\left.30{ }^{\circ} \mathrm{C}\right), 4 \mu \mathrm{l}$ aliquots were transferred to a master microtiter plate well containing $156 \mu \mathrm{l}$ of TB medium $+2 \%$ glc + Amp200, cultured on a plate shaker $\left(8 \mathrm{~h}, 150\right.$ r.p.m., $\left.30{ }^{\circ} \mathrm{C}\right)$ and stored at $4{ }^{\circ} \mathrm{C}$ for up to 2 weeks. The remaining cells were pelleted by centrifugation $\left(10 \mathrm{~min}\right.$ at $\left.4600 \times g, 4{ }^{\circ} \mathrm{C}\right)$ and resuspended in $200 \mu \mathrm{l}$ of $\mathrm{TB}$ medium+Amp200+1 mM IPTG to induce protein expression. After $4 \mathrm{~h}$ induction at $25^{\circ} \mathrm{C}$, cells were pelleted by centrifugation $(10 \mathrm{~min}$ at $4600 \times g, 4{ }^{\circ} \mathrm{C}$ ), the cell pellet was resuspended in $1 \mathrm{ml}$ of the lysis buffer described above for $30 \mathrm{~min}$ at $25^{\circ} \mathrm{C}$ and the insoluble fraction was removed by centrifugation $\left(20 \mathrm{~min}\right.$ at $4600 \times g, 4{ }^{\circ} \mathrm{C}$ ). The soluble fraction was further clarified using 96-well MultiScreen HTS NA clearing filter plates (Millipore, Billerica, MA, USA), as described by the manufacturer. Filtered lysates were transferred to Costar non-binding 96-well plates (Corning) and covered with microplate foil (GE Healthcare). Relative expression levels of $\mathrm{scAb}$ proteins in filtered lysate were obtained using a Minifold I Dot Blot System (Whatman, GE Healthcare) by transferring $10 \mu \mathrm{l}$ of filtered lysate diluted with $90 \mu \mathrm{l}$ of PBS to a nitrocellulose membrane following the manufacturer guidelines. Immunological detection of the polyhistidine-tagged scAb proteins immobilized on the nitrocellulose membrane was performed with anti-His HRP conjugate, as previously described. ${ }^{35}$ The scAbs in filtered lysate were analyzed by SPR, as described below.

\section{Surface plasmon resonance}

Antigen-binding kinetics of $\mathrm{scAb}$ proteins in filtered lysate were analyzed by SPR using a Biacore 3000 (GE Healthcare). Approximately 200 response units of $\mathrm{V}$ antigen in $10 \mathrm{mM}$ sodium acetate ( $\mathrm{pH}$ 5.0) were coupled to a CM5 chip (GE Healthcare) by using 1-ethyl-3(3-dimethylaminopropyl) carbodiimide/ $N$-hydroxy succinimide chemistry. Bovine serum albumin was similarly coupled to the chip and used for in-line subtraction. Kinetic analysis was performed in HBS-P (GE Healthcare) at a flow rate of $25 \mu \mathrm{min}^{-1}$ at $25^{\circ} \mathrm{C}$ unless indicated. Samples were injected for $3 \mathrm{~min}$ and dissociation was monitored for $5 \mathrm{~min}$. The surface was regenerated with two $12 \mathrm{~s}$ injections of $50 \mathrm{~mm}$ phosphoric acid. The antigen-binding kinetics of purified monomeric $\mathrm{scAb}$ were determined in HBS-P at a flow rate of $50 \mu \mathrm{min}^{-1}$. Samples were injected for $2 \mathrm{~min}$ and dissociation was monitored for $10 \mathrm{~min}$. The surface was 
regenerated with two 12-s injections of $50 \mathrm{mM}$ phosphoric acid. Four two-fold dilutions of each $\mathrm{Ab}$ beginning at $160 \mathrm{nM}$ were performed in triplicate, double referenced with a blank and globally fit to a 1:1 Langmuir binding model for the calculation of both $k_{\text {on }}$ and $k_{\text {off }}$ using BIAevaluation software (GE Healthcare). ${ }^{37}$

\section{Ad vectors}

The $\mathrm{Ad} \alpha \mathrm{V}$ is a replication-defective human Ad serotype $5, \mathrm{E} 1^{-} \mathrm{E} 3^{-}$gene transfer vector constructed to direct the expression of the heavy and light chains of the anti-V antigen mAb 2C12.4 from a single promoter. ${ }^{1}$ The expression cassette contains ( $5^{\prime}$ to $\left.3^{\prime}\right)$ the cytomegalovirus immediate early promoter/enhancer, the Ab IgG2b heavy chain coding sequence, a four amino acid furin cleavage site, the 24 amino acid self-cleaving 2A peptide, the $\kappa$-light chain coding sequence and the simian virus 40 polyadenylation signal. Ad $\alpha$ V.H8 was constructed by assembling the genes encoding the $V_{H}$ and $V_{K}$ domains of the high-affinity anti- $\mathrm{V}$ antigen $2 \mathrm{C} 12.4$ variant, $\mathrm{H} 8$, by overlap PCR using the Ad $\alpha \mathrm{V}$ IgG2b expression cassette as a template and the resulting fragment was used to replace the $2 \mathrm{C} 12.4$ coding sequence in $\mathrm{Ad} \alpha \mathrm{V}$. $\mathrm{Ad} \alpha \mathrm{PA}$ is a similarly constructed gene transfer vector encoding an unrelated $\mathrm{Ab}(14 \mathrm{~B} 7-1 \mathrm{H})$ against $B$. anthracis PA and was used as a negative control. ${ }^{29} \mathrm{Ad} \alpha \mathrm{V}, \mathrm{Ad} \alpha \mathrm{V} . \mathrm{H} 8$ and $\mathrm{Ad} \alpha \mathrm{PA}$ were produced in human embryonic kidney 293 cells (American Type Culture Collection, Manassas, VA, USA) and purified by centrifugation twice through a $\mathrm{CsCl}$ gradient, as described. ${ }^{38}$ The titer of each recombinant Ad preparation was determined spectrophotometrically and expressed as pu, as described. ${ }^{39}$

\section{Assessment of $A d \alpha \mathrm{V} . \mathrm{H8}$ in vivo}

Male C57BL/6 mice ( $n=13$ per group) (The Jackson Laboratory, Bar Harbor, ME, USA) were housed under specific-pathogen-free conditions and used at 6-8 weeks of age. Mice were administered $\mathrm{Ad} \alpha \mathrm{V}$ or $\mathrm{Ad} \alpha \mathrm{V} . \mathrm{H} 8\left(10^{9}\right.$, $10^{10}$ or $10^{11} \mathrm{pu}$ ) through the intravenous route. Naïve mice or mice injected with $\mathrm{Ad} \alpha \mathrm{PA}\left(10^{11} \mathrm{pu}\right)$ were used as negative controls. Ad vectors were diluted with saline to the specified dose. Serum was collected through the tail vein 3 days after vector administration, centrifuged at $8000 \times g$ for $20 \mathrm{~min}$ and stored at $-20^{\circ} \mathrm{C}$. Anti-V antigen serum titers were assessed by ELISA using flat-bottomed 96-well EIA/RIA plates (Corning) coated with $100 \mu \mathrm{l}$ of $5 \mu \mathrm{g} \mathrm{ml}^{-1}$ recombinant $\mathrm{V}$ antigen in $0.05 \mathrm{M}$ carbonate buffer ( $\mathrm{pH}$ 7.4) overnight at $4{ }^{\circ} \mathrm{C}$. The plates were washed three times with PBS and blocked with $200 \mu \mathrm{l}$ PBS supplemented with $5 \%$ milk for $1 \mathrm{~h}$ at $23{ }^{\circ} \mathrm{C}$. Serial serum dilutions were added to each well and incubated for $1 \mathrm{~h}$ at $23{ }^{\circ} \mathrm{C}$. The plates were washed three times with PBS containing $0.05 \%$ Tween-20. Immunocomplexes were detected using a sheep anti-mouse IgG HRP conjugate (Sigma-Aldrich) applied at a 1:10 000 dilution in PBS supplemented with $1 \%$ milk incubated for $1 \mathrm{~h}$ at $23{ }^{\circ} \mathrm{C}$. The plates were washed four times with PBS containing $0.05 \%$ Tween-20 and once with PBS, developed using $100 \mu \mathrm{l}$ of peroxidase substrate (Bio-Rad, Hercules, CA, USA) incubated for $15 \mathrm{~min}$ at $23{ }^{\circ} \mathrm{C}$ and the reaction quenched by the addition of $100 \mu \mathrm{l}$ of $2 \%$ oxalic acid. The $\mathrm{A}_{415}$ was measured using a microplate reader (Bio-Rad). $\mathrm{Ab}$ titers were calculated based on $\log$ (optical density)-log(dilution) normalized with purified anti-V antigen Abs to account for affinity differences. Mice challenge studies were conducted at The Public Health Research Institute (PHRI) at the International Center for Public Health (Newark, NJ, USA) under biosafety level 3 conditions. Y. pestis CO92 was grown aerobically in heart infusion broth (Becton Dickinson Difco) at $30{ }^{\circ} \mathrm{C}$ and diluted in saline solution for a challenge dose of $2 \times 10^{4}$ colony-forming units, which corresponds to $363 \mathrm{LD}_{50}$. A total of $25 \mu \mathrm{l}$ of bacterial suspension was used for intranasal infection of mice; bacterial dose was controlled by plating on Yersinia selective agar (Oxoid, Hampshire, UK) and counting colonies for colony-forming unit determination. Survival was monitored daily for 14 days.

\section{Statistical analyses}

The data are presented as mean \pm standard error of the mean. Statistical analyses were performed using the non-paired two-tailed Student's $t$-test, assuming equal variance. Survival evaluation was carried out using Kaplan-Meier analysis. Statistical significance was determined at $P<0.1$.

\section{Conflict of interest}

The authors declare no conflict of interest.

\section{Acknowledgements}

This work was supported by a grant from the Clayton Foundation (to GG), a NIH grant U54 AI057158 (to RGC) and a gift from Robert A. Belfer to Support Development of an Antibioterrorism Vaccine (to RGC). We would like to thank Mridula Rani for generating the sequencing encoding the glycine-serine linker for the antibody fragments. We would also like to thank the staff at the Public Health Research Institute for conducting the Y. pestis challenge studies.

\section{References}

1 Sofer-Podesta C, Ang J, Hackett NR, Senina S, Perlin D, Crystal RG et al. Adenovirus-mediated delivery of an anti-V antigen monoclonal antibody protects mice against a lethal Yersinia pestis challenge. Infect Immun 2009; 77: 1561-1568.

2 Smiley ST. Immune defense against pneumonic plague. Immunol Rev 2008; 225: 256-271.

3 Brouqui P, Raoult D. Arthropod-borne diseases in homeless. Ann N Y Acad Sci 2006; 1078: 223-235.

4 Zhou D, Han Y, Yang R. Molecular and physiological insights into plague transmission, virulence and etiology. Microbes Infect 2006; 8: 273-284.

5 Hill J, Eyles JE, Elvin SJ, Healey GD, Lukaszewski RA, Titball RW et al. Administration of antibody to the lung protects mice against pneumonic plague. Infect Immun 2006; 74: 3068-3070.

6 Hill J, Copse C, Leary S, Stagg AJ, Williamson ED, Titball RW et al. Synergistic protection of mice against plague with monoclonal antibodies specific for the F1 and V antigens of Yersinia pestis. Infect Immun 2003; 71: 2234-2238.

7 Hill J, Leary SE, Griffin KF, Williamson ED, Titball RW. Regions of Yersinia pestis $\mathrm{V}$ antigen that contribute to protection against plague identified by passive and active immunization. Infect Immun 1997; 65: 4476-4482.

8 Maynard JA, Maassen CB, Leppla SH, Brasky K, Patterson JL, Iverson $\mathrm{BL}$ et al. Protection against anthrax toxin by recombinant 
antibody fragments correlates with antigen affinity. Nat Biotechnol 2002; 20: 597-601.

9 Wu H, Pfarr DS, Johnson S, Brewah YA, Woods RM, Patel NK et al. Development of motavizumab, an ultra-potent antibody for the prevention of respiratory syncytial virus infection in the upper and lower respiratory tract. J Mol Biol 2007; 368: 652-665.

10 Marks JD. Deciphering antibody properties that lead to potent botulinum neurotoxin neutralization. Mov Disord 2004; 19 (Suppl 8): S101-S108.

11 Barbas 3rd CF, Hu D, Dunlop N, Sawyer L, Cababa D, Hendry $\mathrm{RM}$ et al. In vitro evolution of a neutralizing human antibody to human immunodeficiency virus type 1 to enhance affinity and broaden strain cross-reactivity. Proc Natl Acad Sci USA 1994; 91: 3809-3813.

12 Mabry R, Rani M, Geiger R, Hubbard GB, Carrion Jr R, Brasky K et al. Passive protection against anthrax by using a high-affinity antitoxin antibody fragment lacking an $\mathrm{Fc}_{\mathrm{c}}$ region. Infect Immun 2005; 73: 8362-8368.

13 Riaño-Umbarila L, Juárez-González VR, Olamendi-Portugal T, Ortíz-León M, Possani LD, Becerril B et al. A strategy for the generation of specific human antibodies by directed evolution and phage display. An example of a single-chain antibody fragment that neutralizes a major component of scorpion venom. FEBS J 2005; 272: 2591-2601.

14 Rajpal A, Beyaz N, Haber L, Cappuccilli G, Yee H, Bhatt RR et al. A general method for greatly improving the affinity of antibodies by using combinatorial libraries. Proc Natl Acad Sci USA 2005; 102: 8466-8471.

15 Harvey BR, Georgiou G, Hayhurst A, Jeong KJ, Iverson BL, Rogers GK et al. Anchored periplasmic expression, a versatile technology for the isolation of high-affinity antibodies from Escherichia coli-expressed libraries. Proc Natl Acad Sci USA 2004; 101: 9193-9198.

16 Hayhurst A, Happe S, Mabry R, Koch Z, Iverson BL, Georgiou G et al. Isolation and expression of recombinant antibody fragments to the biological warfare pathogen Brucella melitensis. J Immunol Methods 2003; 276: 185-196.

17 Hayhurst A. Improved expression characteristics of single-chain Fv fragments when fused downstream of the Escherichia coli maltose-binding protein or upstream of a single immunoglobulin-constant domain. Protein Expr Purif 2000; 18: 1-10.

18 Martin AC. Accessing the Kabat antibody sequence database by computer. Proteins 1996; 25: 130-133.

19 Foote J, Eisen HN. Kinetic and affinity limits on antibodies produced during immune responses. Proc Natl Acad Sci USA 1995; 92: 1254-1256.

20 Mohamed N, Clagett M, Li J, Jones S, Pincus S, D'Alia G et al. A high-affinity monoclonal antibody to anthrax protective antigen passively protects rabbits before and after aerosolized Bacillus anthracis spore challenge. Infect Immun 2005; 73: 795-802.

21 Fang J, Qian JJ, Yi S, Harding TC, Tu GH, VanRoey M et al. Stable antibody expression at therapeutic levels using the 2A peptide. Nat Biotechnol 2005; 23: 584-590.

22 Jiang M, Shi W, Zhang Q, Wang X, Guo M, Cui Z et al. Gene therapy using adenovirus-mediated full-length anti-HER-2 antibody for HER-2 overexpression cancers. Clin Cancer Res 2006; 12: 6179-6185.

23 BenAmmar-Ceccoli S, Humblot S, Crouzier R, Acres B, Kieny MP, Herlyn $\mathrm{D}$ et al. Recombinant vaccinia viruses expressing immuno- globulin variable regions efficiently and selectively protect mice against tumoral B-cell growth. Cancer Gene Ther 2001; 8: 815-826.

24 Daugherty AL, Mrsny RJ. Formulation and delivery issues for monoclonal antibody therapeutics. Adv Drug Deliv Rev 2006; 58: 686-706.

25 Samaranayake H, Wirth T, Schenkwein D, Räty JK, Ylä-Herttuala S. Challenges in monoclonal antibody-based therapies. Ann Med 2009; 41: 322-331.

26 Chung $\mathrm{CH}$. Managing premedications and the risk for reactions to infusional monoclonal antibody therapy. Oncologist 2008; 13: 725-732.

27 Joshi A, Bauer R, Kuebler P, White M, Leddy C, Compton P et al. An overview of the pharmacokinetics and pharmacodynamics of efalizumab: a monoclonal antibody approved for use in psoriasis. J Clin Pharmacol 2006; 46: 10-20.

28 Boyer JL, Kobinger G, Wilson JM, Crystal RG. Adenovirus-based genetic vaccines for biodefense. Hum Gene Ther 2005; 16: 157-168.

29 De BP, Hackett NR, Crystal RG, Boyer JL. Rapid/sustained antianthrax passive immunity mediated by co-administration of Ad/AAV. Mol Ther 2008; 16: 203-209.

30 Hashimoto M, Boyer JL, Hackett NR, Wilson JM, Crystal RG. Induction of protective immunity to anthrax lethal toxin with a nonhuman primate adenovirus-based vaccine in the presence of preexisting anti-human adenovirus immunity. Infect Immun 2005; 73: 6885-6891.

31 Lazar GA, Dang W, Karki S, Vafa O, Peng JS, Hyun L et al. Engineered antibody $F_{C}$ variants with enhanced effector function. Proc Natl Acad Sci USA 2006; 103: 4005-4010.

32 Chiuchiolo MJ, Boyer JL, Krause A, Senina S, Hackett NR, Crystal RG et al. Protective immunity against respiratory tract challenge with Yersinia pestis in mice immunized with an adenovirus-based vaccine vector expressing V antigen. J Infect Dis 2006; 194: 1249-1257.

33 Chen G, Hayhurst A, Thomas JG, Harvey BR, Iverson BL, Georgiou $\mathrm{G}$ et al. Isolation of high-affinity ligand-binding proteins by periplasmic expression with cytometric screening (PECS). Nat Biotechnol 2001; 19: 537-542.

34 Mazor Y, Van Blarcom T, Iverson BL, Georgiou G. E-clonal antibodies: selection of full-length $\mathrm{IgG}$ antibodies using bacterial periplasmic display. Nat Protoc 2008; 3: 1766-1777.

35 Ribnicky B, Van Blarcom T, Georgiou G. A scFv antibody mutant isolated in a genetic screen for improved export via the twin arginine transporter pathway exhibits faster folding. J Mol Biol 2007; 369: 631-639.

36 Fromant M, Blanquet S, Plateau P. Direct random mutagenesis of gene-sized DNA fragments using polymerase chain reaction. Anal Biochem 1995; 224: 347-353.

37 Myszka DG. Improving biosensor analysis. J Mol Recognit 1999; 12: $279-284$

38 Rosenfeld MA, Yoshimura K, Trapnell BC, Yoneyama K, Rosenthal ER, Dalemans W et al. In vivo transfer of the human cystic fibrosis transmembrane conductance regulator gene to the airway epithelium. Cell 1992; 68: 143-155.

39 Mittereder N, March KL, Trapnell BC. Evaluation of the concentration and bioactivity of adenovirus vectors for gene therapy. J Virol 1996; 70: 7498-7509.

$40 \mathrm{Wu} \mathrm{H}$, Pfarr DS, Tang Y, An LL, Patel NK, Watkins JD et al. Ultrapotent antibodies against respiratory syncytial virus: effects of binding kinetics and binding valence on viral neutralization. J Mol Biol 2005; 350: 126-144.

Supplementary Information accompanies the paper on Gene Therapy website (http://www.nature.com/gt) 